\section{Cahiers d'ethnomusicologie}

Anciennement Cahiers de musiques traditionnelles

$7 \mid 1994$

Esthétiques

\title{
Cambodge: Musiques de l'exil
}

L'orchestre de la Troupe de danse classique khmère (1992)

\section{Catherine Geach}

Traducteur : Isabelle Schulte-Tenckhoff

\section{OpenEdition}

\section{Journals}

Édition électronique

URL : http://journals.openedition.org/ethnomusicologie/1477

ISSN : 2235-7688

Éditeur

ADEM - Ateliers d'ethnomusicologie

Édition imprimée

Date de publication : 31 décembre 1994

Pagination : 296-298

ISBN : 2-8257-0503-9

ISSN : 1662-372X

Référence électronique

Catherine Geach, «Cambodge: Musiques de l'exil », Cahiers d'ethnomusicologie [En ligne], 7 | 1994, mis en ligne le 03 janvier 2012, consulté le 21 avril 2019. URL : http://journals.openedition.org/ ethnomusicologie/1477

Ce document a été généré automatiquement le 21 avril 2019.

Tous droits réservés 


\title{
Cambodge : Musiques de l'exil
}

L'orchestre de la Troupe de danse classique khmère (1992)

\author{
Catherine Geach
}

Traduction : Isabelle Schulte-Tenckhoff

\section{RÉFÉRENCE}

Cambodge: Musiques de l'exil. L'orchestre de la Troupe de danse classique khmère. Enregistrements : Jean-Daniel Bloesch, Khao-I-Dang, Thailande (1980) et Wheaton, Maryland, USA (1983-84). Texte de présentation : Giovanni Giuriati. 1 CD AIMP XXIV/VDE 698 (1992).

1 Ces enregistrements uniques offrent un choix de musique classique de danse et de musique traditionnelle de divertissement khmères, interprétées avec beaucoup d'entrain et de vitalité par un ensemble cambodgien plutôt hors du commun. Celui-ci réunit en effet aussi bien des professionnels que des amateurs, rescapés des purges culturelles du régime des Khmers Rouges de Pol Pot, à la fin des années soixante-dix.

2 Leur volonté de vivre et de survivre se manifeste dès le début du trott (une danse traditionnelle de mendiant): son rythme, aux contretemps marqués par les tambours samphô et skor, contraste fortement avec les sons des instruments à cordes et à vent, et des autres percussions (tro sao, klö̈, roneat ek). Toute la danse est animée d'une fougue à couper le souffle.

3 C'est là une bonne introduction pour les auditeurs tant étrangers que cambodgiens; elle leur facilite l'accès à l'univers des Khmers, que sa complexité rend bien souvent hermétique.

4 L'art de la musique traditionnelle khmère se fonde sur un thème sous-jacent évoquant l'harmonie universelle. Chaque instrument évolue dans l'improvisation à partir de cette structure de base - le résultat étant à chaque fois unique. Il est intéressant de constater que, dans le tep monorom (danse classique), le roneat ek (xylophone) a une sonorité et une 
technique d'une grande pureté, tout en recourant à une ornementation imaginative pour conférer une couleur et une substance particulière à la mélodie originelle, alors que la flûte (kloï) reste, à quelques détails près, entièrement fidèle au thème - mais sans le recours à la respiration circulaire traditionnelle, produisant ainsi une sonorité simple et directe, de type occidental. Par ce procédé inhabituel, l'atmosphère éthérée de la danse les danseurs représentent en effet des divinités - se trouve rehaussée. Libre de tout détail encombrant, le son de la flûte s'élève au-dessus de la texture sonore produite par l'orchestre.

5 Un des défis majeurs à relever pour tout orchestre consiste à passer en douceur d'une pièce à l'autre, sans s'arrêter. Dans la musique cambodgienne, notamment celle qui accompagne des événements particuliers ou des cérémonies, de telles transitions sont opérées de façon traditionnelle. Mais même les musiciens familiarisés avec le procédé prennent des risques, pour ainsi dire, quand ils s'apprêtent à changer de rythme, voire de tonalité.

Dans la musique mohori, où diverses pièces se succèdent sans pause, les thèmes littéraires s'enchaînent souvent les uns aux autres. Par exemple, il est courant d'interpréter un chant de tissage (dumbai) avant la pièce se rapportant au tissage des fleurs (krong phka). C'est donc en retenant son souffle qu'on attend, à l'écoute de la quatrième plage, la transition entre deux pièces dont l'association est plutôt inhabituelle : kek mon pi choan et khmer krong phka. Mais le changement est si harmonieux que le moment passe avant que l'on s'en aperçoive, illustrant ainsi à merveille le talent et la profonde attitude de cet orchestre.

7 Ici, les rôles que la flûte et le roneat ek jouent normalement dans l'improvisation sont inversés : c'est au roneat ek d'assumer le rôle "puritain", tandis que la flûte évolue librement autour du thème. Dans ce contexte, on ne peut s'empêcher de remarquer l'absence d'instruments de basse comme le roneat thom (grand xylophone) et le khim thom (instrument à cordes frappées). Cette absence est plutôt surprenante, car ces instruments ne produisent pas seulement un timbre plus grave, mais ils contribuent également à la structure rythmique.

8 L'ensemble compte quelques solistes remarquables, comme les joueurs de kloï (flûte), de roneat ek et de tro sao. Le second trott (pl. 8) met en évidence le talent du joueur de cithare à trois cordes takhé, Phan Bin, qui utilise un instrument difficile mais magnifique pour produire de longues phrases ininterrompues, interprétant ainsi la véritable mélodie de la pièce. C'est aussi un plaisir d'entendre à nouveau la voix traditionnelle khmère, dont le timbre nasal est si puissant qu'il en devient presque envoûtant.

9 Les notices accompagnant les enregistrements, dues à G. Giuriati, un musicologue italien, sont bien présentées et fournissent des données, des descriptions et des photographies fort utiles. Cependant, puisque le CD s'adresse à un public large, il conviendrait de préciser certains points d'intérêt général. Peut-être le texte d'accompagnement ne rendil pas suffisamment explicite le fait que toutes les pièces de la musique cambodgienne évoluent autour d'un thème central, d'un thème clef qu'il s'agit de dégager de l'ornementation et de l'improvisation réalisées par chaque musicien. Une telle précision aiderait l'auditeur à identifier le thème originel et à mieux comprendre les fondements de cette musique. 
10 Il serait également utile de préciser que la musique khmère est antiphonal et de type responsorial. Ainsi l'ensemble mohori suit-il un simple modèle ABA, alors que d'autres orchestres sont susceptibles de recourir à des modèles plus complexes.

11 Bien que les informations fournies dans le texte soient bien documentées et correctes, elles ne vont peut-être pas assez loin du point de vue historique. On oublie souvent que certains aspects de la culture khmère, en particulier ceux qui se rapportent à la musique, remontent au-delà de la célèbre époque d'Angkor (Vat). Il faudrait donc tenir compte d'une période de plus de deux millénaires, car la musique khmère était présente au Cambodge pendant l'ère animiste qui précéda l'influence de l'hindouisme et du bouddhisme. La culture et la musique khmères se distinguent ainsi par une profondeur et une signification historiques particulières, notamment en ce qui concerne les rapports du Cambodge avec ses voisins du Sud-Est asiatique.

12 On est profondément touché par ce disque de musique khmère dont les interprètes sont des réfugiés - les enregistrements ayant été réalisés en partie dans le camp de Khao-IDang, triste endroit situé sur la frontière entre la Thaïlande et le Cambodge, et en partie en exil aux États-Unis. C'est un disque d'une grande importance; figurant parmi les premiers enregistrements réalisés après 1979, il témoigne de la résurgence de la culture traditionnelle khmère, tout en reflétant le courage et la détermination extraordinaires des Cambodgiens à maintenir leur identité dans l'adversité. 